\title{
Does implant design and surgical technique improve the clinical outcome in total knee arthroplasty?
}

\author{
Roland Becker • Michael T. Hirschmann • \\ Jon Karlsson
}

Published online: 1 July 2014

(C) Springer-Verlag Berlin Heidelberg 2014

Recently, a new generation of prostheses for total knee arthroplasty (TKA) were introduced by different companies in order to improve the range of motion, clinical outcome and finally patient satisfaction. However, the basic principles in designing implants for total knee arthroplasty have not changed much over the last two decades. We still need to resect the anterior cruciate ligament (cruciate retaining, CR TKA) or remove both the anterior and posterior cruciate ligaments (posterior stabilised, PS TKA). The femoral component is designed to optimise patella tracking and to unload the patello-femoral compartment. Single and multiple radius designs are being used for the femoral condyles, both showing good reasons for preference. The multi-radius design of the femoral component respects the anatomical shape of the femoral condyles and the physiological change of flexion/extension axis during knee motion. However, CR mobile bearing TKA influence the flexion/extension axis as shown by Iacono et al. [10] in a study published in the current issue. The single-radius design presumes a fixed axis of flexion/ extension, which lies slightly posterior to the natural one. A slight posterior placed axis increases the quadriceps

\footnotetext{
R. Becker $(\bowtie)$

Department of Orthopedics and Trauma Surgery, City Hospital Brandenburg, Hochstrasse 26, 14770 Brandenburg, Germany

e-mail: roland_becker@yahoo.de

M. T. Hirschmann

Department of Orthopaedic Surgery and Traumatology, Kantonsspital Baselland-Bruderholz, Bruderholz, Switzerland e-mail: michael.hirschmann@unibas.ch

J. Karlsson

Department of Orthopaedics, Sahlgrenska University Hospital, Molndal, Sweden

e-mail: jon.karlsson@telia.com
}

moment arm and improves the efficiency of quadriceps muscle function [16]. In clinical practise, better range of motion and less pain, in particular, when rising from a sitting position has been observed with the single-radius design [14]. These findings are in harmony with a biomechanical study by Ostermeier and Stukenborg-Colsman. However, other studies did not show any differences in range of motion or clinical outcome between the singleand multiple radius designs [7]. Both concepts raise the questions whether one should restore anatomy as exactly as possible or as the osteoarthritic knee's anatomy is altered by the disease process anyway and hence one should aim for the best knee function and modify the components accordingly?

What about CR or PS designs in TKA? A Cochrane analysis showed a slightly better range of motion of $8^{\circ}$ using the PS design in comparison to the CR design $\left(105^{\circ}\right.$ vs. $113^{\circ}$ ) [11]. This finding is supported by a number of studies, but the clinical relevance of the differences in maximal degree of flexion of $8^{\circ}$ remains questionable [5]. The prevalence of knee flexion of more than $90^{\circ}$ was studied during a period of $35.7 \mathrm{~h}$ in 21 patients [9]. The patients flexed their knee more than $90^{\circ}$ on average $10 \mathrm{~min}$ during this period only and eight oft them more than $120^{\circ}$ for $2.2 \mathrm{~min}$ only. Range of motion during daily activity depends on patient's activity, but it shows that small improvement in the degree of motion will not affect patients' outcome, but may instead increase the risk of aseptic loosening.

Innovations in terms of the tibial component have been very limited. Increasing the number of component sizes and the re-introduction of asymmetric tibial component are considered to improve the knee function. Posterolateral impingement of the popliteus tendon is rather unlikely when using the asymmetric plateau due to the smaller 
antero-posterior dimension on the lateral compartment in comparison with the medial one [12]. It might be an advantage when using the asymmetric tibial component. The tibial plateau coverage was studied in six different implants [19]. The average coverage of the plateau was $80 \%$, but the asymmetric tibia component showed significantly higher coverage. The study also showed that $49 \%$ of the tibial plateaus had a posterolateral overhang.

The posterior slope helps to improve the maximal flexion in CR design as shown in a cadaver study [4]. The results shown in the cadaver model were not reproduced in the clinical setting, however. Femorotibial gap measurements were taken during surgery at $0^{\circ}, 10^{\circ}, 45^{\circ}, 90^{\circ}$ and $135^{\circ}$ of knee flexion [15]. The authors did not find any correlation between increased slope and range of motion using the CR design. Increased flexion was seen with increasing gap size in the PS design only.

Other factors such as the function of the posterior cruciate ligament might be of greater importance than solely changes of the slope in CR TKA. However, it reveals the controversy in TKA and the difficulty to identify the most crucial parameter for optimal outcome. Good knee function, clinical outcome and patient's satisfaction depend on several factors. The interaction of different aspects is even more difficult to analyse. Most of our studies focus on one aspect only as mentioned earlier, such as the radius of the femoral condyle, CR or PS design or the slope of the tibial component. Focusing on one aspect only means neglecting the majority of most of the relevant aspects in TKA. Clearly, this might be one of the major reasons why many studies do not show any major differences in terms of the clinical outcome and patient satisfaction after TKA.

Besides the development in implant design increased attention has also been paid to the surgical technique. The instrumentation has improved constantly. New instruments, cutting guides and other devices for improving alignment have been developed in order to place the components as accurately as possible. Computer assisted surgery and the recently introduced patient-specific instrumentation (PSI) are increasingly recognised and many surgeons are currently using these new techniques. Again, higher accuracy and reproducibility in component placement with less outliers have been shown when using these techniques. However, there is no proof yet that either computer assisted surgery or patient-specific instrumentation improve clinical outcome [2,6].

One of the key questions remains unanswered. Should we try to mimic the bony anatomy and knee kinematics as close as possible with the new implants or should we rather design an optimal implant, which allows the best function and clinical outcome after TKA. Currently, there appears to be a trend to be as anatomical as possible with the implants.
However, if a more anatomical design and restoration of anatomy is the solution for better outcome in TKA, why do hinged prosthesis with their inherent high degree of constraint and unnatural knee kinematic present very good mid- to long-term results and survival rates as well [1, 17]. This phenomenon puts the finger onto the unsolved burning question how close we actually need to mimic natural knee kinematics?

We are aware that there is only a weak correlation between osteoarthritic changes on radiographs and patients' symptoms. Some patients present severe osteoarthritic changes, but minimal symptoms and other patients show mild degeneration only, while they complain about severe pain and low function. The lack of strong correlation between the anatomical findings and patient satisfaction might also be an issue after TKA. Patient-related factors might play a much more important role than has been assumed in the past. It is well known that the general health status, patient expectation, social integration and mental status have major impact on patient satisfaction after TKA [3, 8, 13, 18, 20].

Improvement in the outcome of TKA remains a highly complex issue, but there is no doubt that arthroplasty is one of the most successful innovations in orthopedics. A successful TKA reduces pain and inactivity during daily life.

\section{References}

1. Baker P, Critchley R, Gray A et al (2014) Mid-term survival following primary hinged total knee replacement is good irrespective of the indication for surgery. Knee Surg Sports Traumatol Arthrosc 22:599-608

2. Barrett W, Hoeffel D, Dalury D et al (2014) In-vivo alignment comparing patient specific instrumentation with both conventional and computer assisted surgery (CAS) instrumentation in total knee arthroplasty. J Arthroplasty 29:343-347

3. Becker R, Döring C, Denecke A et al (2011) Expectation, satisfaction and clinical outcome of patients after total knee arthroplasty. Knee Surg Sports Traumatol Arthrosc 19:1433-1441

4. Bellemans J, Robijns F, Duerinckx J et al (2005) The influence of tibial slope on maximal flexion after total knee arthroplasty. Knee Surg Sports Traumatol Arthrosc 13:193-196

5. Bercik MJ, Joshi A, Parvizi J (2013) Posterior cruciate-retaining versus posterior-stabilized total knee arthroplasty: a meta-analysis. J Arthroplasty 28:439-444

6. Cip J, Widemschek M, Luegmair M et al (2014) Conventional versus computer-assisted technique for total knee arthroplasty: a minimum of 5-year follow-up of 200 patients in a prospective randomized comparative trial. J Arthroplasty. doi:10.1016/j.arth. 2014.04.037

7. Hall J, Copp SN, Adelson WS et al (2008) Extensor mechanism function in single-radius vs multiradius femoral components for total knee arthroplasty. J Arthroplasty 23:216-219

8. Hirschmann MT, Testa E, Amsler F et al (2013) The unhappy total knee arthroplasty (TKA) patient: higher WOMAC and lower KSS in depressed patients prior and after TKA. Knee Surg Sports Traumatol Arthrosc 21:2405-2411 
9. Huddleston JI, Scarborough DM, Goldvasser D et al (2009) 2009 Marshall Urist Young Investigator Award: how often do patients with high-flex total knee arthroplasty use high flexion? Clin Orthop Relat Res 467:1806-1898

10. Iacono F, Bruni D, Bignozzi S et al (2014) Does total knee arthroplasty modify flexion axis of the knee? Knee Surg Sports Traumatol Arthrosc. doi:10.1007/s00167-014-3054-x

11. Jacobs WC, Clement DJ, Wymenga AB (2005) Retention versus sacrifice of the posterior cruciate ligament in total knee replacement for treatment of osteoarthritis and rheumatoid arthritis. Cochrane Database Syst Rev CD004803

12. Kazakin A, Nandi S, Bono J (2014) Diagnosis and treatment of intraoperative popliteus tendon impingement. J Knee Surg. doi:10.1055/s-0034-1367729

13. Lingard EA, Riddle DL (2007) Impact of psychological distress on pain and function following knee arthroplasty. J Bone Joint Surg Am 89:1161-1169

14. Mahoney OM, McClung CD, dela Rosa MA et al (2002) The effect of total knee arthroplasty design on extensor mechanism function. J Arthroplasty 17:416-421
15. Oka S, Matsumoto T, Muratsu H et al (2013) The influence of the tibial slope on intra-operative soft tissue balance in cruciateretaining and posterior-stabilized total knee arthroplasty. Knee Surg Sports Traumatol Arthrosc. doi:10.1007/s00167-013-2535-7

16. Ostermeier S, Stukenborg-Colsman C (2011) Quadriceps force after TKA with femoral single radius. Acta Orthop 82:339-343

17. Steckel H, Klinger HM, Baums MH et al (2005) Long-term results of the blauth knee prosthesis-current status of hinged knee prostheses. Z.Orthop. Ihre Grenzgeb 143:30-35

18. Walton MJ, Newman JH (2008) Pre-operative mental wellbeing and the outcome of knee replacement. Knee 15:277-280

19. Wernecke GC, Harris IA, Houang MT et al (2012) Comparison of tibial bone coverage of 6 knee prostheses: a magnetic resonance imaging study with controlled rotation. J Orthop Surg 20:143-147

20. Yoo JH, Chang CB, Kang YG et al (2011) Patient expectations of total knee replacement and their association with sociodemographic factors and functional status. J Bone Joint Surg $\mathrm{Br}$ 93:337-344 